\title{
Low-Dissipation Silicon Tuning Fork Gyroscopes for Rate and Whole Angle Measurements
}

\author{
Alexander A. Trusov, Member, IEEE, Igor P. Prikhodko, Student Member, IEEE, Sergei A. Zotov, Member, IEEE, \\ and Andrei M. Shkel, Senior Member, IEEE
}

\begin{abstract}
We report a new family of ultra high-Q silicon microelectromechanical systems (MEMS) tuning fork gyroscopes demonstrating angular rate and, for the first time, rate integrating (whole angle) operation. The novel mechanical architecture maximizes the $\mathrm{Q}$-factor and minimizes frequency and damping mismatches. We demonstrated the vacuum packaged SOI dual and quadruple mass gyroscopes with $Q$-factors of 0.64 and 0.86 million at $2 \mathrm{kHz}$ operational frequency, respectively. Due to the stiffness and damping symmetry, the quadruple mass gyroscope was instrumented to measure the angle of rotation directly, eliminating the bandwidth and dynamic range limitations of conventional MEMS vibratory rate gyroscopes. The technology may enable silicon micromachined devices for inertial guidance applications previously limited to precision-machined quartz hemispherical resonator gyroscopes.
\end{abstract}

Index Terms-Energy dissipation, microelectromechanical systems (MEMS) gyroscope, quality factor, rate integrating, vacuum packaging, whole angle.

\section{INTRODUCTION}

$\mathbf{T}$ HE OPERATION of micromachined vibratory gyroscopes is based on a transfer of energy between two modes of vibration caused by the Coriolis effect [1]. Maximization of the drive- and sense-mode quality factors (Q-factors) is key to enhancing performance of micromachined vibratory gyroscopes [2]. Mode-matched silicon tuning fork gyroscopes with Q-factors on the order of 100 thousand have been demonstrated to achieve sub-degree-per-hour bias stability in a $0.1 \mathrm{~Hz}$ measurement bandwidth [3]. However, mode matching of a high-Q vibratory gyroscope instrumented for rate measurements in the open loop fundamentally limits the linear range to below $100 \%$ s and bandwidth to less than $1 \mathrm{~Hz}$ (for an operational frequency below $20 \mathrm{kHz}$ ). These constrains are typically eliminated by operating the rate measuring gyroscope in the closed loop, or force-rebalance mode [4]. An alternative approach is to operate a high-Q mode-matched MEMS gyroscope in the rate integrating, or whole angle mode [5].

Manuscript received February 01, 2011; revised May 16, 2011; accepted May 24, 2011. Date of publication June 23, 2011; date of current version October 21, 2011. This work was supported by the Office of Naval Research and Naval Surface Warfare Center Dahlgren Division under Grant N00014-09-1-0424. The associate editor coordinating the review of this paper and approving it for publication was Dr. Thomas Kenny.

The authors are with the Department of Mechanical and Aerospace Engineering, MicroSystems Laboratory, University of California, Irvine, CA 92697 USA (e-mail: atrusov@uci.edu; iprikhod@uci.edu; szotov@uci.edu; ashkel@uci.edu).

Color versions of one or more of the figures in this paper are available online at http://ieeexplore.ieee.org.

Digital Object Identifier 10.1109/JSEN.2011.2160338
An ideal angle measuring gyroscope, such as the classical macroscale Foucault pendulum, is based on a highly isotropic resonator. The successful realization of a micromachined implementation requires a mechanical structure with highly symmetric mode-shapes providing matched frequency and Q-factors over a practical temperature range. Since the operation of an angle measuring gyroscope is based on the free precession of the inertial mass [1], maximization of the energy dissipation time constant is also critical. Previously reported MEMS gyroscopes, however, suffer from mismatches in frequency and damping, and short energy dissipation constants of less than $1 \mathrm{~s}(0.1 \mathrm{~s}$ typical $)$ making them unfavorable for angle measuring operation.

An optimal MEMS gyroscope architecture for precision rate and angle measurements should comprise symmetric mechanical structure with a combination of high Q-factors, high Coriolis coupling, drive- and sense-mode degeneracy, and frequency tuning capability [6]. These requirements are typically addressed by using a continuous structure, such as micromachined disk or ring [6], [7]. Maximization of energy dissipation time constant for such structures is challenging due to the inherent coupling between operational frequency and Q-factor. An alternative, lumped mass design approach was first introduced in [8], and later the feasibility of interchangeable rate and angle measurements was demonstrated in [9].

Recently, the lumped, four quadrant symmetric architecture was proposed in [10] to address the limitations of the continuous structures. The proposed family of vacuum packaged, dynamically balanced silicon tuning fork gyroscopes achieving free vibrations decay time constants on the order of several minutes, enables interchangeable operation in rate and whole angle modes, Fig. 1. Dissipation of energy through the substrate is minimized by the means of complete antiphase operation facilitated by an integrated mechanical lever mechanism [11]. Operation at low frequencies (on the order of several $\mathrm{kHz}$ ) decouples the mechanical resonance and thermal relaxation time constants, thereby increasing the fundamental thermoelastic Q-factor limit above 1 million.

In this paper, we present a more complete account of the new designs evaluated in [12], as well as experimental characterization of the vacuum sealed quadruple mass gyroscope with improved structural and rate response characteristics. Section II introduces new mechanical architectures of the dual and quadruple mass gyroscopes. In Section III, we discuss the sensors dynamics and signal conditioning for the interchangeable rate and whole angle measurements. Fabrication of the prototypes and their structural, thermal, and rate/angle response 


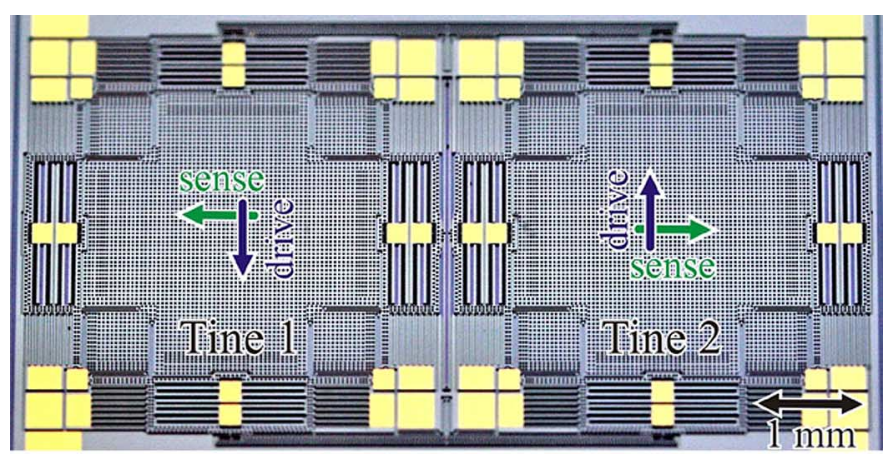

(a)

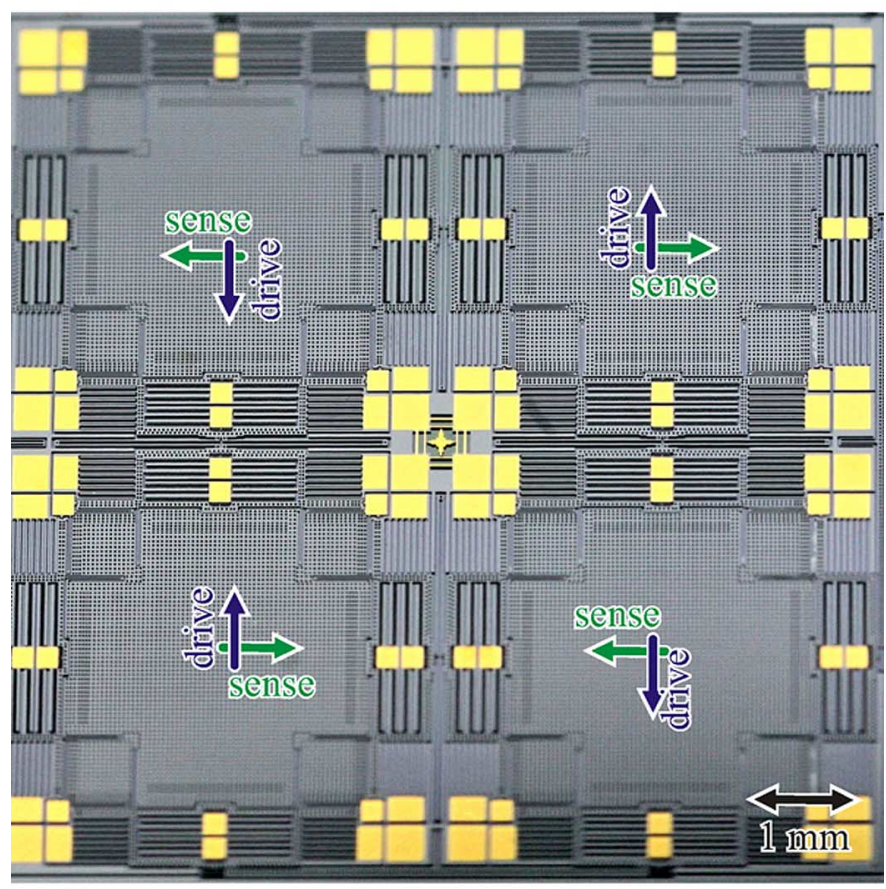

(b)

Fig. 1. Family of ultra-high Q-factor silicon MEMS tuning fork gyroscopes fabricated using a silicon-on-insulator substrate with $100 \mu \mathrm{m}$ thick device layer. The dimensions of each mass are 2.7 by $2.7 \mathrm{~mm}$. The minimum feature size is $5 \mu \mathrm{m}$. (a) Photograph of a fabricated dual mass gyroscope. The chip dimensions are approximately 8.6 by $4.4 \mathrm{~mm}$. (b) Photograph of a fabricated quadruple mass gyroscope. The chip dimensions are 8.6 by $8.6 \mathrm{~mm}$.

characterization are introduced in Section IV, followed by a discussion, in Section $\mathrm{V}$, on the tradeoff between rate and whole angle modes of operation. Section VI concludes the paper with a summary of results.

\section{DESIGN CONCEPT}

In this section, we describe the mechanical architectures of the antiphase operated dual and quadruple mass gyroscopes.

\section{A. Dual Mass Gyroscope}

The mechanical architecture of the dual mass gyroscope, Fig. 1(a), comprises two identical symmetrically decoupled tines [13], a drive-mode antiphase synchronization lever mechanism [11], and coupling flexures for the linear antiphase sense-mode. Each tine is an isotropic resonator consisting

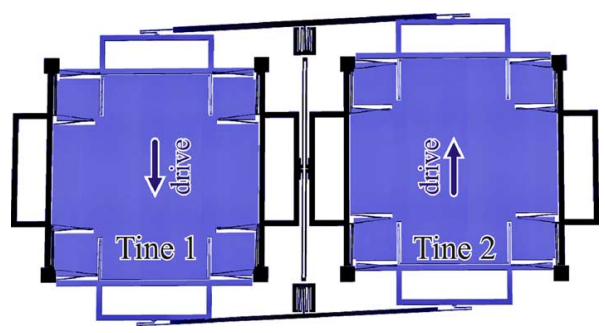

Fig. 2. Dual mass gyroscope operation: levered drive-mode with antiphase synchronization and linear momentum balancing, modeling.

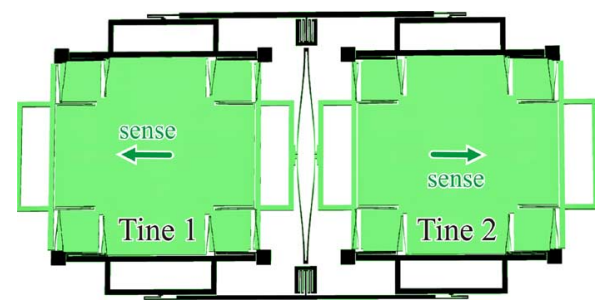

Fig. 3. Dual mass gyroscope operation: linearly coupled antiphase sense-mode with complete dynamic balance for $\mathrm{Q}$ maximization, modeling.

of a square proof mass suspended by two drive-mode and two sense-mode decoupling shuttles. The drive-mode of the dual mass gyroscope is formed by the two tines forced into anti-parallel, antiphase motion synchronized by the integrated mechanical lever system, Fig. 2. The sense-mode of the dual mass gyroscope is formed by the two linearly coupled tines moving in antiphase, Fig. 3.

For rate measuring mode of operation, the gyroscope is continuously driven into antiphase motion using lateral comb electrodes on the drive-mode shuttles. Rotation around the sensitive $z$ axis results in Coriolis excitation of the linear antiphase sensemode, which is detected capacitively using differential parallel plate electrodes on the sense-mode decoupling shuttles. Unlike conventional tuning fork gyroscopes, this dual mass architecture prioritizes the quality factor of the sense-mode by mechanical design. While the intentional mode arrangement maximizes the angular rate sensitivity, the inherent nonsymmetry of dual mass gyroscopes limits the ability to match drive- and sense-mode Q factors in vacuum, as required for the rate integrating (whole angle) mode of operation. The symmetry is achieved by extending the design to the quadruple mass architecture.

\section{B. Quadruple Mass Gyroscope}

Similarly to the dual mass device, the mechanical structure of the quadruple mass gyroscope, Fig. 1(b), comprises four identical symmetrically decoupled tines, a pair of antiphase synchronization lever mechanisms for both the drive- and the sensemode, and linear coupling flexures between the tines. The architecture of the quadruple mass gyroscope builds upon the dual mass design and can be thought of as two levered dual mass gyroscopes coupled together with additional linear flexures and antiphase lever mechanisms. Operation of the quadruple mass gyroscope is illustrated by the Finite-Element Modeling of the drive-mode in Fig. 4. Due to symmetry of the device, the driveand sense-modes are degenerate and spatially oriented at $90^{\circ}$ angle. 


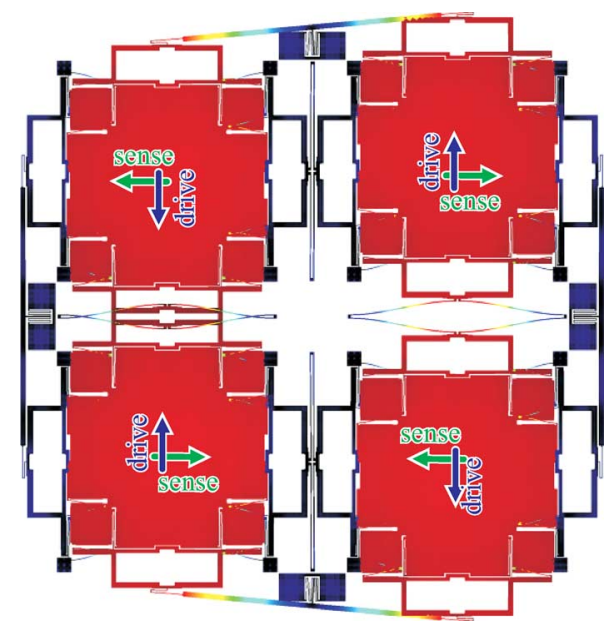

Fig. 4. Quadruple mass gyroscope operation: degenerate, antiphase drive- and sense-modes provide complete dynamic balance, low dissipation of energy, and matching of frequency and Q-factor by mechanical design.

The quadruple mass gyroscope preserves and expands the structural advantages of the levered dual mass design. Unlike conventional tuning fork devices, the levered quadruple mass architecture provides true mechanical rejection of external vibrations and mechanical shocks along both the drive and sense axes. Structural symmetry of the device suggests improved robustness to fabrication imperfections and temperature induced frequency drifts, which are known sources of drifts in high-Q vibratory gyroscopes. Most importantly, the quadruple mass design is expected to enable rate integrating (whole angle) mode of operation due to its unique combination of low energy dissipation and isotropy of both the resonant frequency and damping.

\section{SENSORS DyNAMICS AND INSTRUMENTATION}

In this section, we describe gyroscope dynamics, signal conditioning and control for the angular rate and the rate integrating (whole angle) modes of operation. For the same mechanical element, the signal processing differs depending upon the rate or whole angle mode of operation, Figs. 5 and 6 . The dynamic analysis below assumes that gyroscopes are 2-D isotropic massspring-damper systems with the natural frequency $\omega$ and the quality factor $Q$.

\section{A. Angular Rate Mode}

In the conventional angular rate mode of operation, the $z$ axis gyroscope is continuously driven by a force $f_{x} \sin (\omega t)$ into a steady-state resonant motion in the drive direction ( $x$ axis). The driving voltages are imposed across the differential lateral comb electrodes of the drive-mode shuttles. The rotation around the $z$ axis induces the motion in the sense direction ( $y$ axis), with the amplitude proportional to an input angular rate $\Omega$. Sense-mode vibrations are detected using differential parallel plate electrodes of the sense-mode shuttles. For the open-loop operation (at slow rates), the amplitude of $y$ motion is small compared to $x$ amplitude, and the equations of motion are (including the centrifugal force):

$$
\begin{aligned}
& \ddot{x}+\frac{\omega}{Q} \dot{x}+\left(\omega^{2}-\Omega^{2}\right) x=f_{x} \sin (\omega t) \\
& \ddot{y}+\frac{\omega}{Q} \dot{y}+\left(\omega^{2}-\Omega^{2}\right) y=-2 \dot{x} \Omega .
\end{aligned}
$$

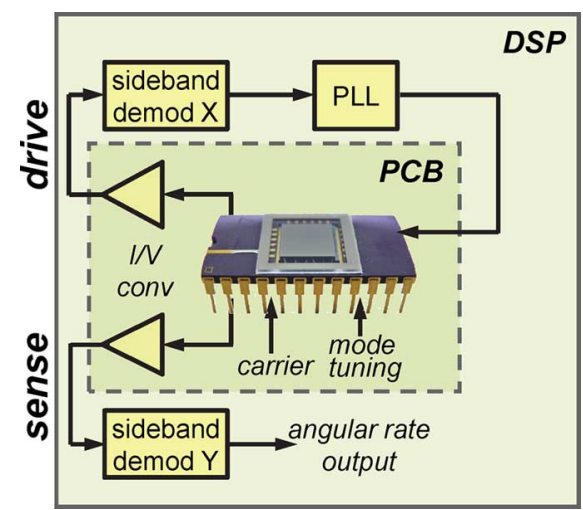

Fig. 5. Gyroscope signal conditioning and control for the rate performance characterization (rate mode of operation).

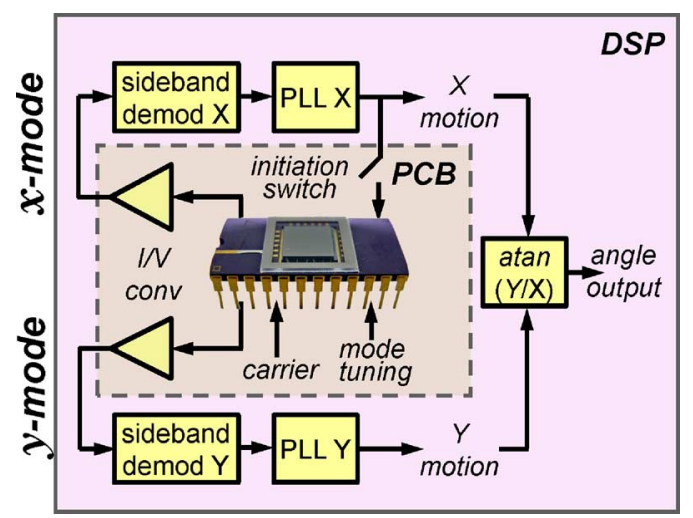

Fig. 6. Gyroscope signal conditioning and control for the direct angle measurements (whole angle mode of operation).

The input rate is obtained by demodulating the sense-mode output ( $y$ motion) with the reference signal $\cos (\omega t)$, Fig. 5. Fig. 7 shows input-output relationship of an open loop gyroscope instrumented in rate measuring mode (simulation). Simulation model assumes $2 \mathrm{kHz}$ operational frequency and presence of the centrifugal force. The response of the gyroscope is linear for a limited input range, determined by the ratio $\omega /(2 Q)$. The operation in the whole angle mode is expected to eliminate this constraint.

\section{B. Whole Angle Mode}

The whole angle mode is based upon the classical Foucault pendulum operation, where the vibration axis is allowed to precess freely in response to the inertial rotation (including the centrifugal force)

$$
\begin{aligned}
& \ddot{x}+\frac{\omega}{Q} \dot{x}+\left(\omega^{2}-\Omega^{2}\right) x=2 \dot{y} \Omega \\
& \ddot{y}+\frac{\omega}{Q} \dot{y}+\left(\omega^{2}-\Omega^{2}\right) y=-2 \dot{x} \Omega .
\end{aligned}
$$

Unlike the conventional rate measuring mode, where the axis of vibration is locked to the intended drive direction, the whole angle mode is based on free vibrations $\left(f_{x}=0\right)$. To initiate free vibrations, the gyroscope was electrostatically driven into the $x$-mode antiphase resonant motion using a phase-locked loop, which was then abruptly turned off, Fig. 6. During rotation, the free vibrating axis remains fixed in the inertial space, thereby 


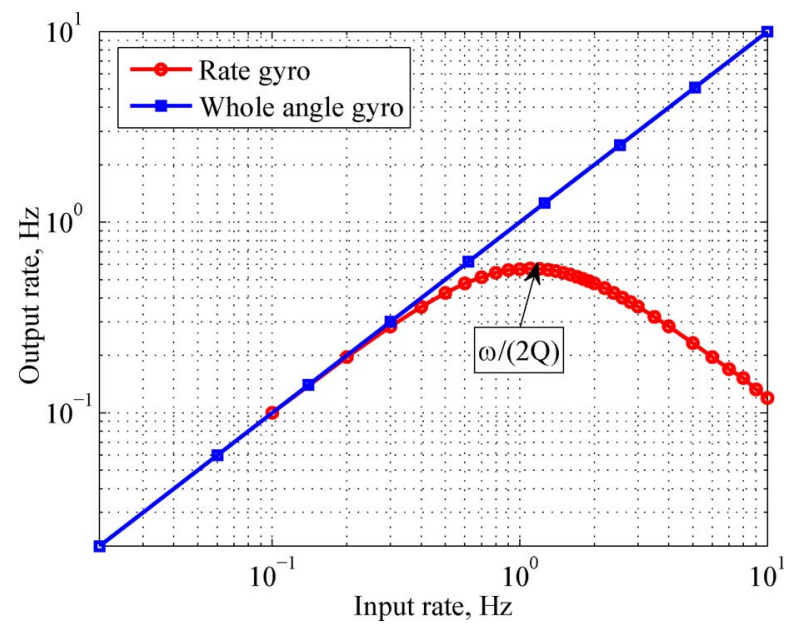

Fig. 7. Input-output relationship of an open loop gyroscope instrumented in rate and whole angle modes. The nonlinearity of the response is observed for the rate operating mode at large input rotations as opposed to the whole angle mode, which is linear in the full range.

providing an orientation reference. The precession angle $\theta$, measured from the amplitudes of $x$ and $y$, motion provides a direct instantaneous measure of the gyroscope rotation [4]

$$
\theta=-\int \Omega d t
$$

The motion of $x$ - and $y$-modes (amplitude and phase) is detected capacitively by synchronous demodulation, and converted to an angle using a geometric relation $\theta=\operatorname{atan}(\|y\| /\|x\|) / 2$. Fig. 7 demonstrates linear response of a $2 \mathrm{kHz}$ resonant gyroscope operated in the whole angle mode (simulation). The fundamentally unlimited input range is enabled by free precession of the vibrating axis as opposed to constrained operation in the rate mode.

\section{EXPERIMENTAL CHARACTERIZATION}

In this section we experimentally evaluate the dual and quadruple mass gyroscope designs using in-house fabricated and vacuum packaged prototypes.

\section{A. Fabrication and Vacuum Packaging}

The fabrication of prototypes was done using an in-house, wafer-level, single-mask process based on silicon-on-insulator (SOI) substrates with $50 \mu \mathrm{m}$ and $100 \mu \mathrm{m}$ thick device layer and a $5 \mu \mathrm{m}$ buried oxide layer. A hard mask was defined on the structural layer by depositing, patterning, and dry etching a $1 \mu \mathrm{m}$ thick surface oxide layer. Devices were defined in the highly doped structural layer by DRIE. Finally, the perforated structures were released using a timed $20 \% \mathrm{HF}$ acid bath. A packagelevel technology for the robust vacuum sealing of the high- $Q$ gyroscopes was implemented to allow experimental characterization of stand-alone sensors, Fig. 8. The packaging procedure comprises the eutectic attachment of a gyroscope die to a ceramic package, wirebonding, and eutectic sealing of the device in a $10^{-6}$ Torr vacuum preceded by activation of the getter deposited on the lid [14].

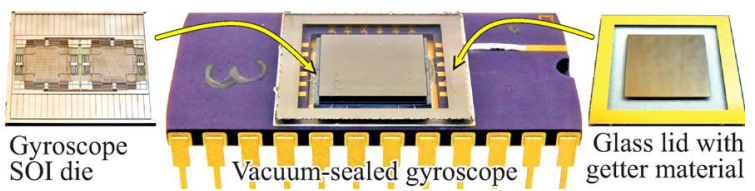

Fig. 8. Photograph of a vacuum packaged gyroscope, showing a gyro die, ceramic DIP-24 package, and custom made glass lid with getter material.

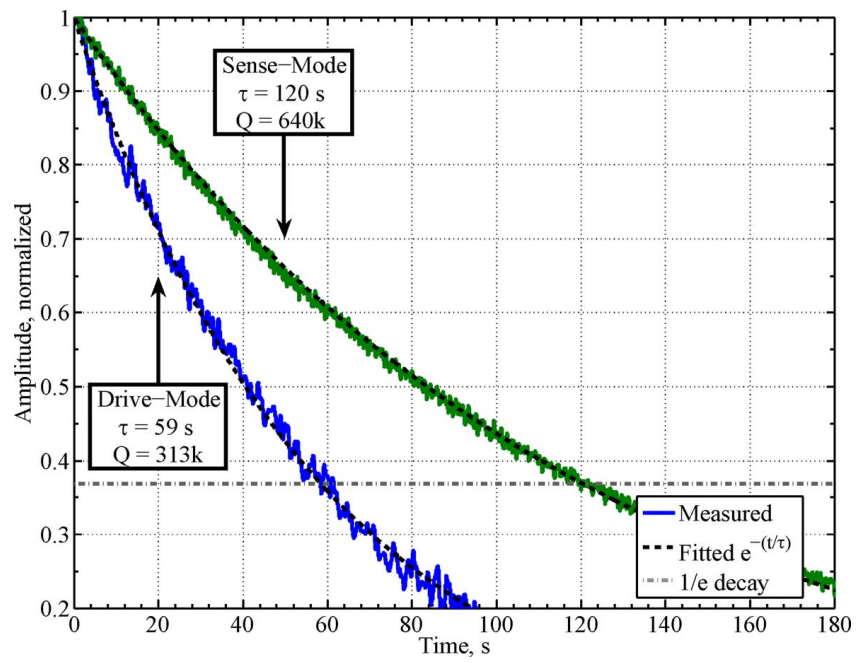

Fig. 9. Measurement of packaged gyroscope Q-factors using ring-down tests. Drive- and sense-mode Q-factor is 0.31 and 0.64 million, respectively.

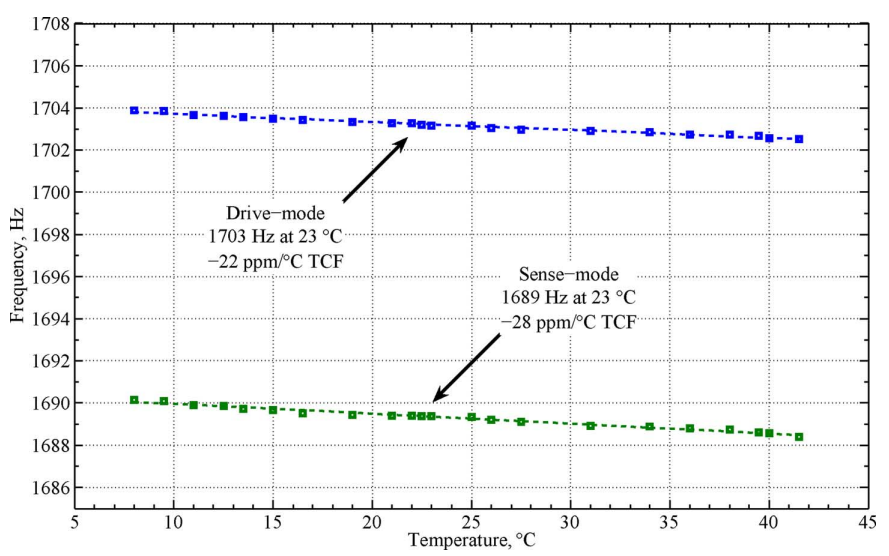

Fig. 10. Measured frequency versus temperature for the vacuum packaged dual mass gyroscope revealed a linear TCF of $-25 \pm 3 \mathrm{ppm} /{ }^{\circ} \mathrm{C}$.

\section{B. Interface Electronics}

The devices are experimentally characterized using PCB electronics connected to the digital signal processing unit, Figs. 5 and 6. The sensors are mounted on a PCB with front-end transimpedance amplifiers. All signal processing is performed in real-time using a FPGA-based lock-in amplifier from Zurich Instruments. The sensor motion is actuated electrostatically and detected capacitively using an Electromechanical Amplitude Modulation (EAM) technique.

\section{Dual Mass Gyroscope Characterization}

Ring-down characterization of a vacuum packaged dual mass gyroscope with a $1.7 \mathrm{kHz}$ drive-mode operational frequency 


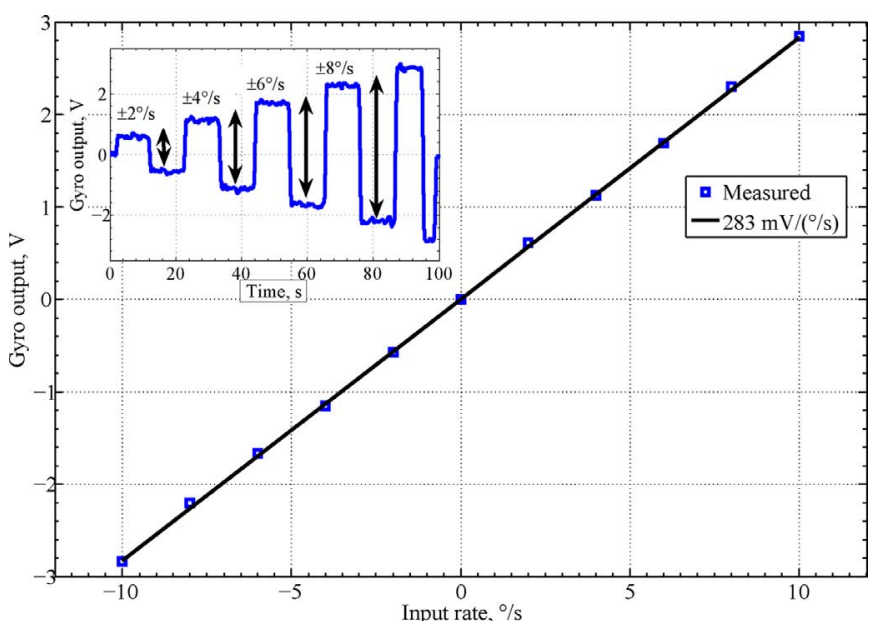

Fig. 11. Experimental rate characterization of the dual mass gyroscope under a $7 \mathrm{~Hz}$ mismatch between the drive- and sense-mode resonances.

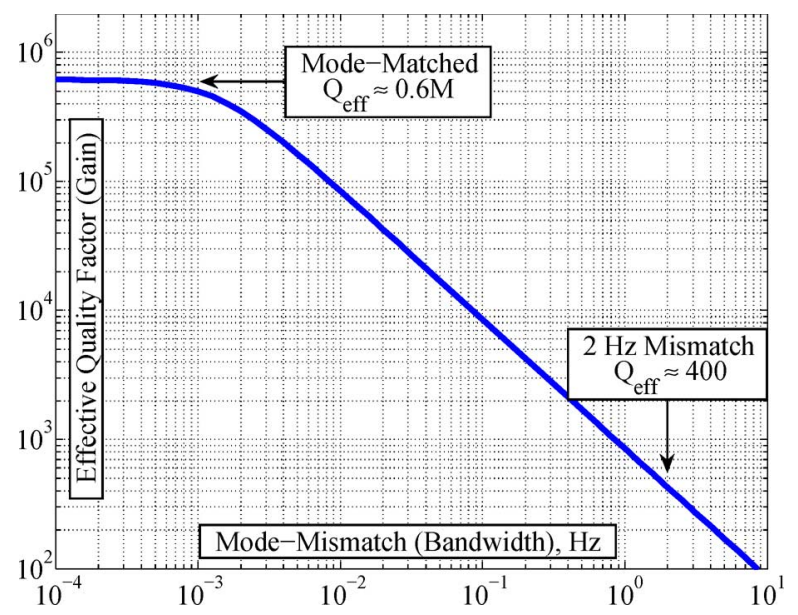

Fig. 12. Analytical modeling of gain-bandwidth characteristic of the dual mass gyroscope. Sensitivity improvement is $+40 \mathrm{~dB}$ for $0.1 \mathrm{~Hz}$ matching (and $+60 \mathrm{~dB}$ for $10 \mathrm{mHz}$ )

revealed a measured drive-mode Q-factor of 0.31 million and a sense-mode Q-factor of 0.64 million, Fig. 9. To achieve the best rate sensitivity, a vacuum packaged gyroscope should be operated under a close matching between the drive- and the sense-modes maintained over a practical range. Fig. 10 shows measured frequency-temperature characteristics of the driveand sense-modes, revealing linear TCFs of $-22 \mathrm{ppm} /{ }^{\circ} \mathrm{C}$ and $-28 \mathrm{ppm} /{ }^{\circ} \mathrm{C}$, respectively. Preliminary open loop rate characterization of the gyroscope was performed with a $7 \mathrm{~Hz}$ frequency mismatch, Fig. 11. From the gain-bandwidth analysis in Fig. 12, the rate sensitivity is expected to improve by $+40 \mathrm{~dB}$ for $0.1 \mathrm{~Hz}$ mode matching, and by $+60 \mathrm{~dB}$ for $0.01 \mathrm{~Hz}$ matching. The dual mass gyroscope experimental results confirm the ultra-high $\mathrm{Q}$ design and motivate the investigation of the completely balanced, symmetric quadruple mass gyroscope.

\section{Quadruple Mass Gyroscope Characterization}

Structural characterization of a standalone vacuum sealed quadruple mass gyroscope with $2,170 \mathrm{~Hz}$ operational frequency is shown in Fig. 13. The ring-down measurements revealed

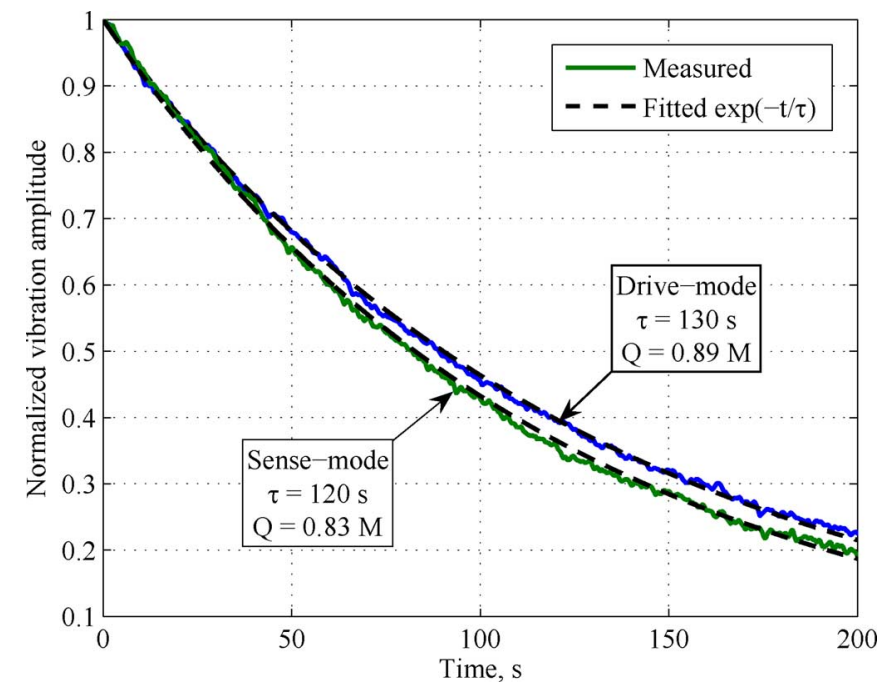

Fig. 13. Measurement of the quadruple mass gyroscope $Q$-factors using ringdown tests. Both drive- and sense-mode Q-factor are close to 0.86 million.

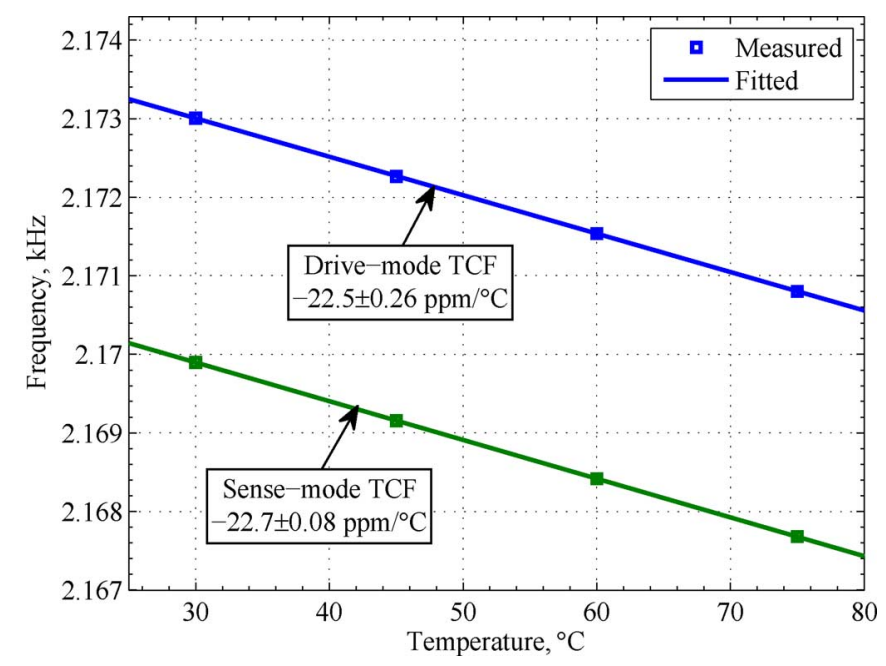

Fig. 14. Measured frequency and $\mathrm{Q}$ versus temperature for the quadruple mass gyroscope. Drive and sense have a precisely matched TCF of $-22.6 \mathrm{ppm} /{ }^{\circ} \mathrm{C}$.

virtually identical drive- and sense-mode Q-factor of approximately 0.86 million, yielding an isotropic energy dissipation time constant in excess of $2 \mathrm{~min}$. The effect of temperature on the drive- and sense-mode frequency and Q-factor was characterized using a thermal chamber, Fig. 14. The experiments revealed a linear dependency of the drive- and sense-mode frequencies on temperature with a closely matched TCF of -22.5 and $-22.7 \mathrm{ppm} /{ }^{\circ} \mathrm{C}$ with an uncertainty of $\pm 0.26 \mathrm{ppm} /{ }^{\circ} \mathrm{C}$. Isotropic Q-factors above 0.5 million were experimentally observed for temperatures up to $80{ }^{\circ} \mathrm{C}$, Fig. 15. Measurements of Q-factors demonstrated the dependence $\mathrm{Q} \propto 1 / T^{3}$, characteristic of the thermoelastic dissipation (TED) [15], which we believe to be the dominant energy loss mechanism in the vacuum packaged quadruple mass gyroscopes (viscous damping is eliminated by vacuum packaging, while anchor loss is minimized by the balanced design).

The quadruple mass gyroscope was characterized in both angular rate and rate integrating (whole angle) modes of operation. For the angular rate operation, the structure was continuously 


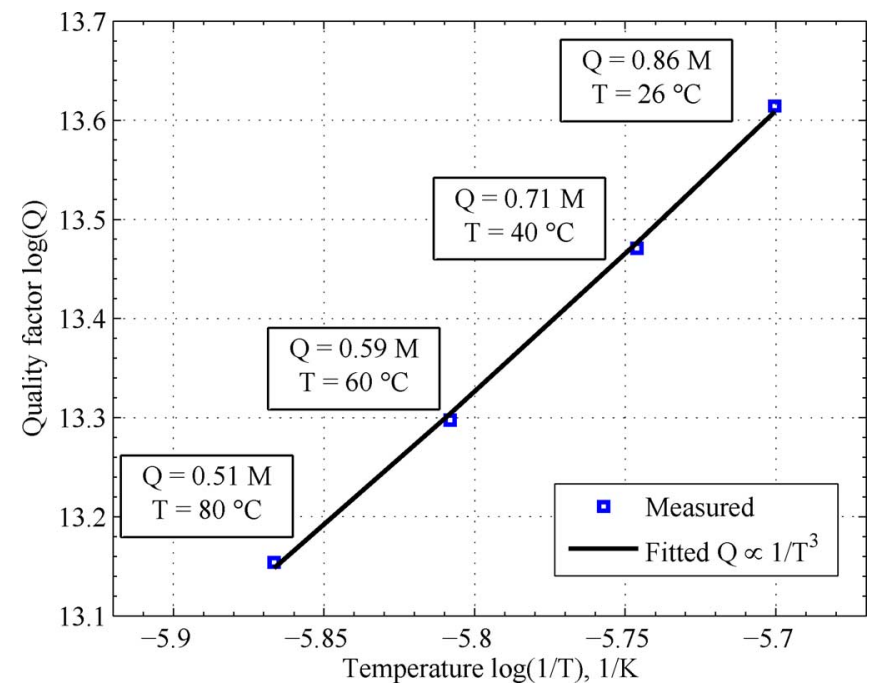

Fig. 15. Measured Q-factors of the quadruple mass gyroscope demonstrating $1 / \mathrm{T}^{3}$ dependence for temperatures from 25 to $80^{\circ} \mathrm{C}$. Q-factors above 0.5 million are observed for temperatures up to $80^{\circ} \mathrm{C}$.

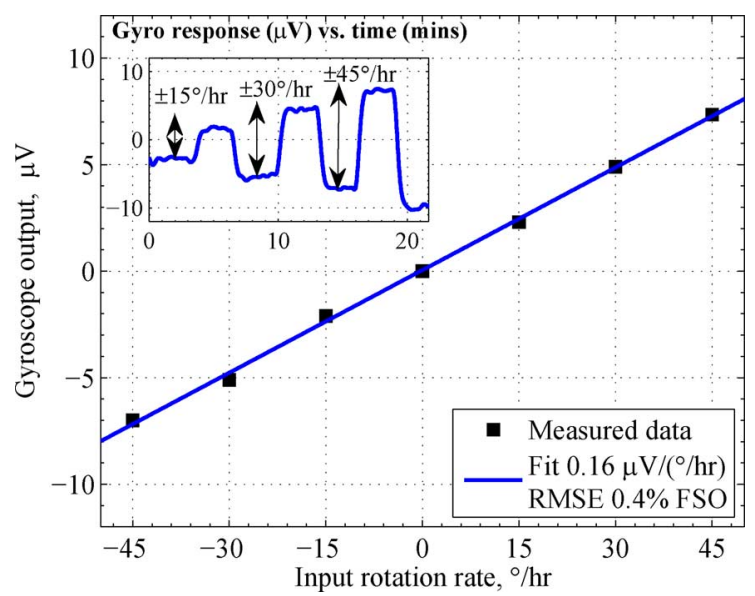

Fig. 16. Experimental rate characterization of the quadruple mass gyroscope under a $0.2 \mathrm{~Hz}$ mismatch between the drive- and sense-mode resonances.

driven into drive-mode antiphase vibrations using an off-chip phase locked loop (PLL), while the Coriolis induced response of the sense-mode was detected to produce a rate output. Rate characterization was performed under a $0.2 \mathrm{~Hz}$ frequency mismatch in the input range of $\pm 45^{\circ} / \mathrm{h}$, Fig. 16. Based on the scale-factor sensitivity analysis presented in Fig. 12, the frequency mismatch of $0.2 \mathrm{~Hz}$ provides $+40 \mathrm{~dB}$ improvement in the rate resolution and ensures robustness to the frequency mismatch variations. In this mode of operation, the quadruple mass gyroscope demonstrated to resolve rotations on the order of $15 \circ / \mathrm{h}$ (approximately Earth's rotation rate). The detailed experimental characterization of the rate measuring mode revealed a $0.9^{\circ} / \mathrm{h}$ bias instability and a $\pm 20^{\circ} / \mathrm{s}$ linear range in open-loop operation of the sense-mode [16].

To experimentally evaluate the rate integrating (whole angle) operation, the vacuum packaged quadruple mass gyroscope was first mode-matched to within $20 \mathrm{mHz}$ and initiated into antiphase drive-mode resonance using an off-chip PLL. Upon achieving amplitude of several micrometers, all the driving

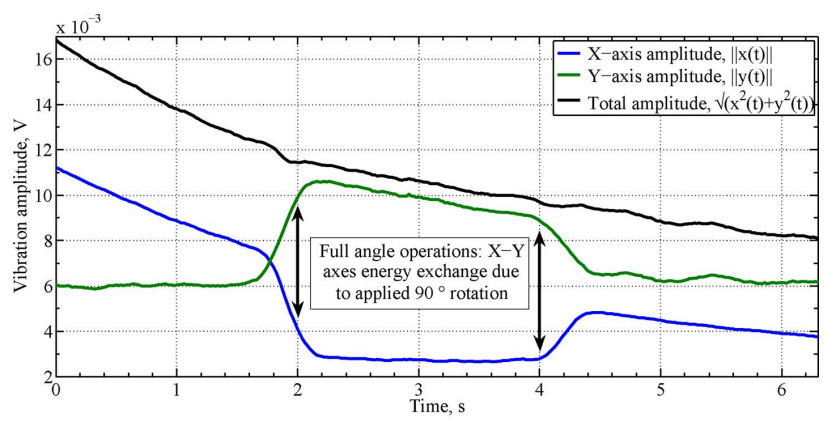

(a)

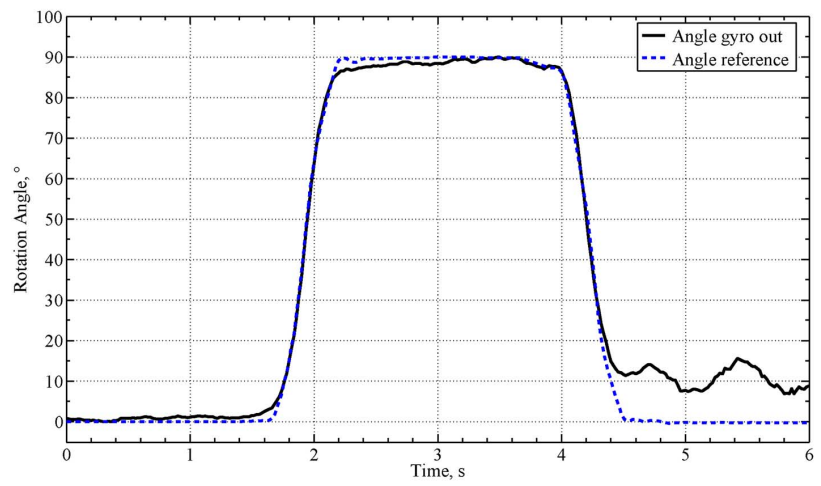

(b)

Fig. 17. Experimental demonstration of the rate integrating (whole angle) operation using free vibrations of the ultra-high $\mathrm{Q}$ four mass gyroscope. (a) Exchange of energy between the two modes in response to $90^{\circ}$ rotations. (b) Angle of rotation measured directly from the proof mass precession.

voltages were switched off to enable free precession of the ultra-high Q isotropic resonator. When a rotation is applied, the orientation of the free vibrations pattern remains fixed in inertial space, providing a direct measurement of the rotation angle. The amplitude change of drive- and sense-modes was recorded in response to a fast $\pm 90^{\circ}$ rotation, Fig. 17(a). The precession angle of a vibration axis was calculated in real time from the vibration amplitudes of drive- and sense-modes, and compared to the angle of input rotation (reference), Fig. 17(b). The detailed experimental characterization of the mode-matched quadruple mass device operated in the angle measuring mode confirmed $\pm 450^{\circ} / \mathrm{s}$ linear range in a $100 \mathrm{~Hz}$ bandwidth with RMS error of $0.4 \%$ FSO [5].

\section{DISCUSSION}

In this section, we highlight tradeoffs between rate and whole angle operating modes. The choice of a gyroscope operating mode depends upon the application requirements. Rate operating mode provides high precision measurements of slow motion and low-noise characteristics at the expense of relatively reduced bandwidth, range, and scale factor stability. In contrast, the whole angle mode provides fundamentally unlimited measurement bandwidth and linear input range. The angular gain factor of an angle gyroscope is also extremely stable and defined purely by the mechanical structure. However, the angle noise typically limits the gyroscope resolution [17]. The proposed family of low-dissipation tuning fork gyroscopes enables interchangeable rate and whole angle operation, making one high- $\mathrm{Q}$ mechanical structure suitable for high precision and wide range measurements. 


\section{CONCLUSION}

We reported a family of silicon MEMS tuning fork gyroscopes, demonstrating the interchangeable operation in angular rate and rate integrating (whole angle) regimes. A vacuum packaged dual mass SOI gyroscope with a $1.7 \mathrm{kHz}$ operational frequency demonstrated drive- and sense- mode Q-factors of 0.3 and 0.6 million, respectively. A completely symmetric, dynamically balanced quadruple mass gyroscope with a $2.2 \mathrm{kHz}$ operational frequency demonstrated virtually identical driveand sense-mode Q factors of 0.9 million. The low-energy dissipation allows reducing the driving voltages to $1 \mathrm{mV}$ ac (with $1 \mathrm{~V} \mathrm{dc}$ polarization), and provides mechanical sensitivity on the order of $1 \mathrm{~nm} /(\% / \mathrm{h})$ with a fundamental mechanical-thermal resolution limit on the order of $0.01^{\circ} / \mathrm{h} / \sqrt{\mathrm{Hz}}[18]$. The measured frequency-temperature characteristics of the gyroscopes are linear with closely matched TCFs, making them more suitable for electrostatic compensation than quadratic dependency of high-Q quartz devices [19]. Unlike most high-Q silicon devices, the gyroscopes can also be ovenized [20], without a significant performance sacrifice since Q-factors on the order of 0.5 million are sustained up to $80{ }^{\circ} \mathrm{C}$. Due to the stiffness and damping symmetry, the quadruple mass gyroscope can be instrumented to measure the angle of rotation directly, eliminating the bandwidth and dynamic range limitations of conventional MEMS vibratory rate gyroscopes. The technology may enable silicon micromachined devices for inertial guidance applications previously limited to precision-machined quartz hemispherical resonator gyroscopes.

\section{ACKNOWLEDGMENT}

The authors would like to acknowledge T. Lee and J. Zendejas of the UCLA Nanoelectronics Research Facility for assistance with the fabrication of prototypes, Dr. F. Heer from Zurich Instruments AG for assistance with signal processing, H. Florence of SAES Getters and P. Barnes of SST International for assistance with vacuum packaging, and I. Chepurko for assistance with the interface electronics. The gyroscopes were designed and characterized at the MicroSystems Laboratory, University of California, Irvine.

\section{REFERENCES}

[1] A. M. Shkel, "Type I and Type II micromachined vibratory gyroscopes," in Proc. IEEE/ION Position Locat. Navigat. Symp., San Diego, CA, Apr. 24-27, 2006, pp. 586-593.

[2] M. Weinberg, R. Candler, S. Chandorkar, J. Varsanik, T. Kenny, and A. Duwel, "Energy loss in MEMS resonators and the impact on inertial and RF devices," in Proc. Int. Conf. Solid State Sens. Actuators Microsyst., Denver, CO, Jun. 21-25, 2009, pp. 688-695.

[3] M. Zaman, A. Sharma, Z. Hao, and F. Ayazi, "A mode-matched silicon-yaw tuning-fork gyroscope with subdegree-per-hour allan deviation bias instability," J. Microelectromech. Syst., vol. 17, no. 6, pp. 1526-1536, Dec. 2008.

[4] D. Lynch, "Coriolis vibratory gyros," in Proc. Symp. Gyro Technol., Stuttgart, Germany, Sep. 15-16, 1998, pp. 1.0-1.14.

[5] I. Prikhodko, S. Zotov, A. Trusov, and A. Shkel, "Foucault pendulum on a chip: Angle measuring silicon MEMS gyroscope," in Proc. IEEE Int. Conf. Micro-Electro-Mechan. Syst., Cancun, Mexico, Jan. 23-27, 2011, pp. 161-164.
[6] K. Shcheglov, "DRG - A high performance MEMS gyro," in Proc. Joint Precision Azimuth Sensing Symp., Las Vegas, NV, Aug. 2-4, 2010.

[7] M. W. Putty, "A micromachined vibrating ring gyroscope," Ph.D. dissertation, Univ. Michigan, Ann Arbor, MI, 1995.

[8] A. M. Shkel and R. T. Howe, "Micro-Machined Angle Measuring Gyroscope," U.S. Patent 6481 285, Nov. 19, 2002.

[9] C. Painter, "Micromachined vibratory gyroscopes with imperfections," Ph.D. dissertation, Univ. California, Irvine, CA, 2005.

[10] A. A. Trusov, A. R. Schofield, and A. M. Shkel, "Micromachined tuning fork gyroscopes with ultra-high sensitivity and shock rejection," U.S. Application 20100313657.

[11] A. Trusov, A. Schofield, and A. Shkel, "Gyroscope architecture with structurally forced anti-phase drive-mode and linearly coupled antiphase sense-mode," in Proc. Int. Conf. Solid State Sens. Actuators Microsyst., Denver, CO, Jun. 21-25, 2009, pp. 660-663.

[12] A. Trusov, I. Prikhodko, S. Zotov, A. Schofield, and A. Shkel, "Ultrahigh Q silicon gyroscopes with interchangeable rate and whole angle modes of operation," in Proc. IEEE Sensors Conf., Waikoloa, HI, Nov. 1-4, 2010, pp. 864-867.

[13] M. Kranz and G. Fedder, "Micromechanical vibratory rate gyroscopes fabricated in conventional CMOS," in Proc. Symp. Gyro Technol., Stuttgart, Germany, Sep. 16-17, 1997, pp. 3.0-3.8.

[14] A. Schofield, A. Trusov, and A. Shkel, "Versatile vacuum packaging for experimental study of resonant mems," in Proc. IEEE Int. Conf. Micro-Electro-Mechan. Syst., Hong Kong, China, Jan. 24-28, 2010, pp. 516-519.

[15] B. Kim, M. Hopcroft, R. Candler, C. Jha, M. Agarwal, R. Melamud, S. Chandorkar, G. Yama, and T. Kenny, "Temperature dependence of quality factor in MEMS resonators," J. Microelectromech. Syst., vol. 17 , no. 3, pp. $755-766$, Jun. 2008

[16] I. Prikhodko, S. Zotov, A. Trusov, and A. Shkel, "Sub-degree-per-hour silicon MEMS rate sensor with 1 million Q-factor," in Proc. 16th Int. Conf. Solid-State Sens., Actuators, Microsyst. (TRANSDUCERS'11), Beijing, China, Jun. 5-9, 2011, pp. 2809-2812.

[17] D. M. Rozelle, "The hemispherical resonator gyro: From wineglass to the planets (AAS 09-176)," in Proc. 19th AAS/AIAA Space Flight Mechanics Meeting, Feb. 2009, pp. 1157-1178.

[18] A. Trusov, A. Schofield, and A. Shkel, "Micromachined rate gyroscope architecture with ultra-high quality factor and improved mode ordering," Sens. Actuators A: Phys., pp. 26-34, 2010.

[19] Y. Xie, S.-S. Li, Y.-W. Lin, Z. Ren, and C.-C. Nguyen, "1.52-GHz micromechanical extensional wine-glass mode ring resonators," IEEE Trans. Ultrasonics, Ferroelectrics and Frequency Control, vol. 55, no. 4, pp. 890-907, Apr. 2008.

[20] S.-H. Lee, J. Cho, S. Lee, M. Zaman, F. Ayazi, and K. Najafi, "A low-power oven-controlled vacuum package technology for high-performance MEMS," in Proc. IEEE Int. Conf. Micro-Electro-Mechan. Syst., Sorrento, Italy, Jan. 25-29, 2009, pp. 753-756.

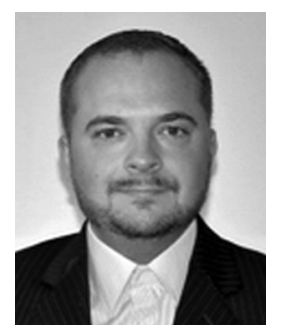

Alexander A. Trusov (M'09) received the B.S. and M.S. degrees in applied mathematics and mechanics from the Moscow State University, Moscow, Russia, in 2004 , and the M.S. and Ph.D. degrees in mechanical and aerospace engineering from the University of California, Irvine, in 2006 and 2009, respectively.

$\mathrm{He}$ is currently a Project Scientist at the UC Irvine MicroSystems Laboratory, where he serves as a PI and Co-PI on a variety of federally sponsored programs pursuing high-performance inertial microsystems. He has published over 40 journal and conference papers on MEMS and inertial sensors, has seven pending U.S. patents, and serves as a reviewer for major journals in the fields of MEMS and sensors. His research interests include design, modeling, fabrication, and vacuum packaging of micromachined inertial systems, design of characterization experiments, and statistical data processing and analysis.

Dr. Trusov is a member of the American Society of Mechanical Engineers (ASME). He is a recipient of an Outstanding Paper Award on low-dissipation silicon MEMS gyroscopes at the Transducers 2011 Conference. 


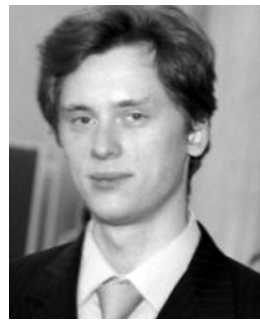

Igor P. Prikhodko (S'09) received the B.S. and M.S. degrees (cum laude) in applied mathematics and mechanics from the Moscow State University, Moscow, Russia, in 2007, and the M.S. degree in mechanical and aerospace engineering from the University of California, Irvine, in 2008. Currently, he is working towards the $\mathrm{Ph} . \mathrm{D}$. degree at the MicroSystems Laboratory, University of California, Irvine.

His primary research focus is the full-cycle development of inertial micromachined sensors, reflected in 12 journal and conference papers.

Mr. Prikhodko is a recipient of the 2008 Holmes Fellowship Award and the 2011 Outstanding Paper Award at the Transducers Conference. He serves as a reviewer for major MEMS journals.

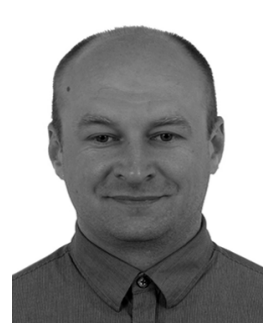

Sergei A. Zotov (M'11) received the M.S. and Ph.D. degrees in mechanical engineering and control systems from the Tula State University, Tula, Russia, in 1999 and 2002, respectively.

$\mathrm{He}$ is currently a Postdoctoral Scientist at the MicroSystems Laboratory, University of California, Irvine, where he is responsible for the design, fabrication, and testing of micromachined devices and systems for inertial navigation. He holds eight Russian patents and three U.S. patents pending. Over the last ten years his focus has been on MEMS accelerometers and gyroscopes.

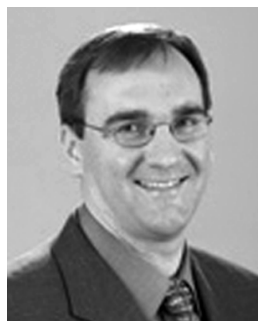

Andrei M. Shkel (SM'11) received the Diploma degree (With Excellence) in mechanics and mathematics from the Moscow State University, Moscow, Russia, in 1991, and the Ph.D. degree in mechanical engineering from the University of Wisconsin, Madison, in 1997.

$\mathrm{He}$ is a Program Manager in the Microsystems Technology Office of the Defense Advanced Research Projects Agency (DARPA), Arlington, VA.

$\mathrm{He}$ is serving in this capacity while on leave from his faculty position as a Professor in the Department of Mechanical and Aerospace Engineering at the University of California, Irvine, where he is also the Director of the UCI Microsystems Laboratory. He is the holder of 15 U.S. and international patents. His professional interests are reflected in more than 120 publications.

Dr. Shkel is a recipient of the 2009 Research Award from the IEEE Sensors Council, the 2006 UCI Research Award, the 2005 NSF CAREER Award, and the 2002 George E. Brown, Jr., Award. He is an Editor of the JournAL OF MiCROELECTROMECHANICAL SYSTEMS (JMEMS). 